\title{
Evaluating perennial ryegrass cultivars: improving testing
}

\author{
G.A. KERR ${ }^{1}$, D. F. CHAPMAN ${ }^{2}$, E. R. THOM ${ }^{3}$, C. MATTHEW ${ }^{4}$, A. VAN DER LINDEN ${ }^{5}$, \\ D. B. BAIRD ${ }^{6}$, E. JOHNSTON ${ }^{7}$ and J. R. CORKRAN ${ }^{1}$ \\ ${ }^{1}$ Agriseeds, 2547 Old West Coast Rd, RD1, Christchurch 7671 \\ ${ }^{2}$ DairyNZ, PO Box 160, Lincoln University 7647 \\ ${ }^{3}$ DairyNZ, Private Bag 3221, Hamilton 3240 \\ ${ }^{4}$ Massey University, Private Bag 11222, Palmerston North 4442 \\ ${ }^{5}$ Centre for Crop Systems Analysis, Wageningen University, P.O. Box 430, 6700 AK, Wageningen, The Netherlands \\ ${ }^{6}$ VSN (NZ) Ltd, 8 Mariposa Crescent, Christchurch 8025 \\ ${ }^{7}$ AsureQuality, PO Box 6, Lincoln University, Lincoln 7647 \\ gak@agriseeds.co.nz
}

\begin{abstract}
The agronomic performance of a range of perennial ryegrass cultivar-endophyte combinations was compared in 16 trials conducted at sites throughout New Zealand. Each trial was run for 3 years according to seed industry evaluation protocols, measuring variables including: dry matter (DM) yield (total annual and seasonal DM yield), ryegrass ground cover at the end of 3 years, susceptibility to plant pulling, and rust incidence. The change in DM yield over the 3-year term of each trial was also analysed. There were significant differences among cultivars in total annual DM yield, and in seasonal DM yield for each of the five periods of the year among which yield was split (winter, early spring, late spring, summer, and autumn). Mean annual yield declined between Year 1 and Year 3 in all regions except Taranaki, by between $0.8 \mathrm{t} \mathrm{DM} /$ ha (Canterbury) and $5.3 \mathrm{t} \mathrm{DM} / \mathrm{ha}$ (Waikato). There were significant region, year, region $\times$ year, and cultivar $\times$ year interactions in yield change. Significant differences in ground cover score for perennial ryegrass at the end of 3 years were recorded among cultivars. However, these differences did not mirror on-farm observations of ryegrass persistence in the Waikato during the drought of 2007/08, suggesting that the standard trial protocols currently used do not adequately test persistence. It is recommended that industry cultivar testing needs to more accurately assess perennial ryegrass persistence, using new protocols including running trials on commercial farms, for more than 3 years, and using mixed swards. Genetic differences in persistence could be generated more quickly by choosing sites known to challenge perennial ryegrass growth and survival.
\end{abstract}

Keywords: perennial ryegrass, cultivar evaluation, DM yield, persistence testing, plant pulling, rust

\section{Introduction}

New Zealand's ability to produce quality livestock product at internationally competitive prices is based on grazing pastures in situ, and the challenge to New Zealand's plant breeding industry is to continually improve pasture production (Clark et al. 2001).

Genetic improvement of pasture plants has been pursued for over 85 years (Wratt \& Smith 1983), with documented gains in dry matter (DM) production, disease resistance and forage quality (Corkill 1949; Kerr 1987; Easton et al. 1989; Easton et al. 1997; Woodfield 1999; Easton et al. 2001). Genetic gains averaging around $0.5 \%$ per year for total annual DM yield (Lee et al. 2012) have been demonstrated through small plot trials (Kerr 1987; Pennell et al. 1990; Easton et al. 2001; Hume et al. 2007).

Total annual DM yield of pasture is a key driver of animal performance in pastoral systems (Williams et al. 2007). The seasonality of supply is equally important, because it strongly influences the balance between feed supply and demand on a month-bymonth basis. Chapman et al. (2012) calculated that the economic value of additional dry matter grown varied for dairy systems in different regions of New Zealand. Extra feed in early spring carried high economic value $(\$ 0.42-\$ 0.48$ per $\mathrm{kg}$ additional DM/ ha) in all regions, whereas the value of summer feed was lower, and varied according to region $(\$ 0.33-\$ 0.40$ per $\mathrm{kg}$ additional $\mathrm{DM} / \mathrm{ha}$ in the Waikato and the lower North Island; \$0.12-\$0.17 per kg additional DM/ha in Canterbury and Southland).

Genetic gains in DM yield identified in plot trials have sometimes proved difficult to capture in animal production trials (Woodward et al. 2001; Crush et al. 2006). However, small plot trials are a practical, relatively low cost way to evaluate pasture cultivars, and remain the dominant source of information for analysing genotype $\times$ environment interactions, and estimating the economic value delivered to farmers through plant breeding (e.g. McEvoy et al. 2011; Bryant et al. 2012). While many such trials have been conducted in New Zealand over several decades, few data from them have 
been published. Yet, these data can reveal considerable information about, for example, the relative agronomic performance of different perennial ryegrass types (diploid versus tetraploid; mid-season flowering versus late-season flowering), and interactions between type and growing environment.

The utility of these trials for assessing other important agronomic traits, such as persistence, has not been considered in any depth. More information is required to clarify the discriminatory power of small plot trials for DM yield, and to determine their value in resolving genotype, endophyte and environmental influences on pasture persistence. This information will help improve the value to the farming industry of pasture cultivar evaluation systems in New Zealand.

This paper presents results from small plot trials conducted throughout New Zealand comparing cultivars and breeding lines of perennial ryegrass (Lolium perenne), the grass of choice for long-term pastures under fertile conditions in New Zealand (Hunt \& Easton 1989; Easton et al. 2011). The variables presented include DM yield, ground cover of ryegrass after 3 years, and resistance to plant pulling and rust. Further analysis was undertaken to assess yield stability over the 3-year trials.

\section{Methods}

\section{Trial programme}

A total of 16 separate perennial ryegrass yield trials, conducted by three different operators, were completed as described in Table 1. Trials were sown in March or April, except those in Southland which were sown in December. For all trials, data were collected from 1 June following sowing through to 31 May, 3 years later. Plots were all maintained as pure ryegrass swards, with herbicides used to control other species. All North Island trials, along with those at Kirwee and Burnham in Canterbury, were rotationally grazed by dairy cows or dairy heifers, whereas the Chertsey, Courtenay and Winton trials were rotationally grazed by sheep. All trials included four replicates (12-15 $\mathrm{m}^{2}$ plot area) of each cultivar or breeding line, typically arranged in a randomised row-column design. Most trials contained 20 entries, which included a range of named cultivars (some with different endophyte strains) and various breeding lines. Some 67 breeding lines were included in statistical analyses, but are omitted from the results reported here, apart from 'LP534' which was later released as the cultivar 'Trojan'.

Trials were conducted according to New Zealand seed industry standards, as prescribed in the National Forage Variety Trial Protocol (NZPBRA 2010). Trial procedures were independently audited by AsureQuality through field visits every year, and final trial results were checked through independent analyses by VSN (NZ) Ltd, with this also audited by AsureQuality.

\section{Measurements and management}

DM yield was assessed on all plots when a mean mass of approximately $3000 \mathrm{~kg} \mathrm{DM} / \mathrm{ha}$ was first reached by one of the entries. Yield was measured by mowing an area of $2.5-3 \mathrm{~m}^{2}$ to $5 \mathrm{~cm}$ height, recording total fresh weight, and then oven-drying a subsample for determination of DM content. The area mown was rotated across three or four discrete non-overlapping positions within each plot. Yield assessment was immediately followed by grazing, after which plots were trimmed, if necessary, to an even post-grazing residual. Grazing was followed by an application of nitrogen-based fertiliser equivalent to approximately $30 \mathrm{~kg} \mathrm{~N} / \mathrm{ha}$. On average each trial was assessed for DM yield 29 times over 3 years.

Results were split into five seasons defined as: winter - June and July; early spring - August and September; late spring - October and November; summer - December to February, and autumn - March to May. Where the growth period for a yield assessment straddled more than one season, the yield was split between seasons based on the proportion of days of growth in each.

Ground cover of perennial ryegrass was assessed at the end of each trial through point analysis (Radcliffe $\&$ Mountier 1964). A total of 100 points per plot were checked to estimate the percentage of ground area covered by perennial ryegrass for each trial entry. Assessment was typically conducted 2 weeks following grazing.

The eight trials grazed by cows or heifers, particularly those at Newstead, often exhibited ryegrass plant pulling. On the 22 occasions when pulling occurred, each trial plot was visually scored on a 1-9 scale, with $9=$ no pulled plants in the plot. In one trial with very high levels of plant pulling, scoring was stopped after 2 years, as several entries had few intact plants left.

High levels of ryegrass infection with crown rust (Puccinia coronata) were observed on seven occasions in five North Island trials. On these occasions, each trial plot was visually assessed and scored on a 1-9 scale for the presence of rust, with $1=$ very high levels of rust and $9=$ no rust.

\section{Data analysis}

To assess stability, or persistency, of DM yield over the 3-year trials, data from the 16 trials were compiled into 909 observations of mean annual yield across trial replicates for each cultivar in each trial, and a factorial ANOVA performed to ascertain yield trends over the 3 -year duration of each trial, regional yield differences, and region $\times$ year and cultivar $\times$ year interactions. 


\section{Results and Discussion \\ DM yield}

There were significant differences among cultivars in total annual and seasonal DM yield in all five seasons analysed (Table 2), with yields similar to those in previous ryegrass cultivar comparison work (Easton et al. 2001). These differences could be expected to translate to sizeable differences in profitability. For example, in autumn, the range between the lowest and highest diploid cultivars was around $600 \mathrm{~kg} \mathrm{DM} / \mathrm{ha}$, or about $\$ 180 / \mathrm{ha}$ if an economic value of $\$ 0.30 \mathrm{per} \mathrm{kg}$ additional $\mathrm{DM} /$ ha is assumed (Chapman et al. 2012).

Diploid and tetraploid cultivars are split in Table 2. This is because tetraploids were likely to be disadvantaged in the trial protocol due to greater animal preference for tetraploids compared to diploids, causing harder grazing (O’Donovan \& Delaby 2005), lower post-grazing residuals, and lower herbage mass at the time of DM yield assessment (which was implemented for all cultivars once the first cultivar in a trial had reached approximately $3000 \mathrm{~kg} \mathrm{DM} / \mathrm{ha}$ ). It is notable that the relative yields of tetraploids were often significantly lower than relative yields of diploids, for example, in early and late spring.

It is also notable that the relative yield of the same cultivar with different endophytes sometimes differed: for example, 'Bronsyn' with NEA6 endophyte ranked significantly higher for total annual DM yield than did 'Bronsyn' with AR1. Endophyte strain effects have been observed in perennial ryegrass for both insect control (Popay \& Hume 2011) and animal feeding preference (Edwards et al. 1993). In these trials run under "cafeteria" grazing it is not possible to determine whether the greater DM yield of a ryegrass-endophyte combination is due to better growth, improved insect resistance, reduced animal preference and therefore

Table 1: Summary of 16 trial sites split by region.

\begin{tabular}{|c|c|c|c|c|c|c|c|}
\hline Region & Sown & Location & $\begin{array}{l}\text { Latitude } \\
\text { Longitude }\end{array}$ & Soil type & $\begin{array}{l}\text { Altitude } \\
\text { a.s.l. }\end{array}$ & $\begin{array}{l}\text { Average } \\
\text { rainfall }\end{array}$ & Operator \\
\hline \multirow[t]{3}{*}{ Waikato } & 2003 & Cambridge & $\begin{array}{c}37^{\circ} 89^{\prime} \mathrm{S} \\
175^{\circ} 43^{\prime} \mathrm{E}\end{array}$ & Kereone silt loam & $60 \mathrm{~m}$ & $1183 \mathrm{~mm}$ & Agriseeds \\
\hline & 2005 & Newstead & $\begin{array}{l}37^{\circ} 78^{\prime} \mathrm{S} \\
175^{\circ} 36^{\prime} \mathrm{E}\end{array}$ & $\begin{array}{l}\text { Te Kowhai peaty } \\
\text { silt loam }\end{array}$ & $40 \mathrm{~m}$ & $1200 \mathrm{~mm}$ & DairyNZ \\
\hline & 2007 & Newstead & $\begin{array}{c}37^{\circ} 78^{\prime} \mathrm{S} \\
175^{\circ} 36^{\prime} \mathrm{E}\end{array}$ & Te Rapa silt loam & $40 \mathrm{~m}$ & $1200 \mathrm{~mm}$ & DairyNZ \\
\hline \multirow[t]{3}{*}{ Taranaki } & 2004 & $\begin{array}{c}\text { TARS, } \\
\text { Whareroa }\end{array}$ & $\begin{array}{l}39^{\circ} 61^{\prime} \mathrm{S} \\
174^{\circ} 31^{\prime} \mathrm{E}\end{array}$ & $\begin{array}{l}\text { Egmont black } \\
\text { loam }\end{array}$ & $90 \mathrm{~m}$ & $1100 \mathrm{~mm}$ & DairyNZ \\
\hline & 2006 & $\begin{array}{c}\text { TARS, } \\
\text { Whareroa }\end{array}$ & $\begin{array}{l}39^{\circ} 61^{\prime} \mathrm{S} \\
174^{\circ} 31^{\prime} \mathrm{E}\end{array}$ & $\begin{array}{l}\text { Egmont black } \\
\text { loam }\end{array}$ & $90 \mathrm{~m}$ & $1100 \mathrm{~mm}$ & DairyNZ \\
\hline & 2008 & $\begin{array}{c}\text { TARS, } \\
\text { Whareroa }\end{array}$ & $\begin{array}{l}39^{\circ} 61^{\prime} \mathrm{S} \\
174^{\circ} 31^{\prime} \mathrm{E}\end{array}$ & $\begin{array}{l}\text { Egmont black } \\
\text { loam }\end{array}$ & $90 \mathrm{~m}$ & $1100 \mathrm{~mm}$ & DairyNZ \\
\hline \multirow[t]{3}{*}{ Manawatu } & 2003 & $\begin{array}{l}\text { Massey } \\
\text { No } 4 \text { dairy }\end{array}$ & $\begin{array}{c}40^{\circ} 40^{\prime} \mathrm{S} \\
175^{\circ} 62^{\prime} \mathrm{E}\end{array}$ & $\begin{array}{l}\text { Tokomaru silt } \\
\text { loam }\end{array}$ & $60 \mathrm{~m}$ & $963 \mathrm{~mm}$ & $\begin{array}{c}\text { Massey } \\
\text { University }\end{array}$ \\
\hline & 2005 & $\begin{array}{l}\text { Massey } \\
\text { No } 1 \text { dairy }\end{array}$ & $\begin{array}{c}40^{\circ} 38^{\prime} \mathrm{S} \\
175^{\circ} 61^{\prime} \mathrm{E}\end{array}$ & $\begin{array}{l}\text { Manawatu silt } \\
\text { loam }\end{array}$ & $34 \mathrm{~m}$ & $963 \mathrm{~mm}$ & $\begin{array}{c}\text { Massey } \\
\text { University }\end{array}$ \\
\hline & 2007 & $\begin{array}{l}\text { Massey } \\
\text { No } 4 \text { dairy }\end{array}$ & $\begin{array}{c}40^{\circ} 40^{\prime} \mathrm{S} \\
175^{\circ} 62^{\prime} \mathrm{E}\end{array}$ & $\begin{array}{l}\text { Tokomaru silt } \\
\text { loam }\end{array}$ & $67 \mathrm{~m}$ & $963 \mathrm{~mm}$ & $\begin{array}{c}\text { Massey } \\
\text { University }\end{array}$ \\
\hline \multirow[t]{5}{*}{ Canterbury } & 2003 & Kirwee & $\begin{array}{l}43^{\circ} 49^{\prime} \mathrm{S} \\
172^{\circ} 19^{\prime} \mathrm{E}\end{array}$ & Chertsey silt loam & $190 \mathrm{~m}$ & $805 \mathrm{~mm}^{\star}$ & Agriseeds \\
\hline & 2003 & Courtenay & $\begin{array}{c}43^{\circ} 46^{\prime} \mathrm{S} \\
172^{\circ} 18^{\prime} \mathrm{E}\end{array}$ & Hatfield silt loam & $190 \mathrm{~m}$ & $805 \mathrm{~mm}^{*}$ & Agriseeds \\
\hline & 2005 & Burnham & $\begin{array}{c}43^{\circ} 60^{\prime} \mathrm{S} \\
172^{\circ} 27^{\prime} \mathrm{E}\end{array}$ & Lismore silt Loam & $150 \mathrm{~m}$ & $670 \mathrm{~mm}^{\star}$ & Agriseeds \\
\hline & 2007 & Chertsey & $\begin{array}{l}43^{\circ} 78^{\prime} \mathrm{S} \\
171^{\circ} 89^{\prime} \mathrm{E}\end{array}$ & Lismore silt Loam & $150 \mathrm{~m}$ & $700 \mathrm{~mm}^{*}$ & Agriseeds \\
\hline & 2008 & Courtenay & $\begin{array}{l}43^{\circ} 46^{\prime} \mathrm{S} \\
172^{\circ} 18^{\prime} \mathrm{E}\end{array}$ & Hatfield silt loam & $190 \mathrm{~m}$ & $805 \mathrm{~mm}^{\star}$ & Agriseeds \\
\hline \multirow[t]{2}{*}{ Southland } & 2007 & Winton & $\begin{array}{c}46^{\circ} 17^{\prime} \mathrm{S} \\
168^{\circ} 33^{\prime} \mathrm{E}\end{array}$ & $\begin{array}{l}\text { Pukemutu silt } \\
\text { loam }\end{array}$ & $50 \mathrm{~m}$ & $1100 \mathrm{~mm}$ & Agriseeds \\
\hline & 2008 & Winton & $\begin{array}{c}46^{\circ} 17^{\prime} \mathrm{S} \\
168^{\circ} 33^{\prime} \mathrm{E}\end{array}$ & $\begin{array}{l}\text { Pukemutu silt } \\
\text { loam }\end{array}$ & $50 \mathrm{~m}$ & $1100 \mathrm{~mm}$ & Agriseeds \\
\hline
\end{tabular}

\footnotetext{
${ }^{*}$ Canterbury trials all irrigated
} 
decreased grazing pressure, or a combination of these factors.

Popay et al. (2003) also observed host genotype $\times$ endophyte strain interactions in perennial ryegrass. Further information is required to understand how these interactions occur, and what they mean for pasture performance.

Although Table 2 provides data on the comparative performance of cultivars, actual DM yields are likely to be underestimated. There are two reasons for this: firstly, non-recording of DM yield accumulated between time of assessments and grazing, particularly if grazing was delayed by stock availability or weather; and secondly, DM losses when plots were trimmed after grazing to ensure consistent post-grazing residuals between plots.

\section{Stability of yield}

The stability, or persistency, of DM yield over time has been questioned (Easton et al. 2011; Parsons et al. 2011). Region, year, and the year $\times$ region interaction effects were all highly significant in the analysis of yield stability from these trials $(\mathrm{P}<0.001)$. On average, Year 3 yield was $1.95 \mathrm{t} \mathrm{DM/ha} \mathrm{lower} \mathrm{than} \mathrm{Year} 1$ yield,

Table 2: $\quad$ Combined analysis of dry matter (DM) yield of diploid and tetraploid cultivars over 16 three-year trials, relative to trial mean $=100 \%$, ranked on total yield. Significance lettering given for $5 \%$ LSD. Cultivars must have been in a minimum of three trials to be included.

\begin{tabular}{|c|c|c|c|}
\hline Entry* & Winter & $\begin{array}{l}\text { Early } \\
\text { Spring }\end{array}$ & $\begin{array}{l}\text { Late } \\
\text { Spring }\end{array}$ \\
\hline
\end{tabular}

\section{Diploid cultivars}

\begin{tabular}{|c|c|c|c|c|c|c|c|c|c|c|c|c|}
\hline Trojan NEA2 & 112 & a & 105 & a & 103 & $\mathrm{ac}$ & 114 & a & 110 & $a b$ & 109 & $\mathrm{a}$ \\
\hline Tolosa NEA2 & 107 & ac & 100 & ag & 108 & a & 109 & ac & 110 & $a b$ & 107 & $a b$ \\
\hline Bronsyn NEA6 & 88 & hi & 101 & af & 105 & $a b$ & 109 & $a b$ & 112 & a & 106 & $a b$ \\
\hline Arrow AR1 & 104 & be & 104 & $\mathrm{ac}$ & 104 & $a b$ & 107 & $\mathrm{bc}$ & 106 & bd & 106 & $a b$ \\
\hline Alto SE & 97 & eg & 98 & $\mathrm{bg}$ & 107 & a & 109 & $a b$ & 108 & ac & 105 & $a b$ \\
\hline Arrow SE & 103 & be & 102 & ad & 103 & $\mathrm{ac}$ & 107 & bc & 105 & bd & 104 & $\mathrm{bc}$ \\
\hline Alto AR1 & 103 & be & 100 & $\mathrm{bg}$ & 104 & $a b$ & 106 & bd & 102 & de & 103 & bc \\
\hline Matrix SE & 104 & be & 97 & $\mathrm{cg}$ & 101 & bd & 107 & $\mathrm{bd}$ & 108 & ac & 103 & bd \\
\hline Extreme AR6 & 100 & ce & 102 & ae & 100 & bd & 104 & bd & 109 & $a b$ & 103 & bd \\
\hline Extreme AR37 & 103 & be & 105 & a & 100 & bd & 101 & $c f$ & 104 & be & 103 & bd \\
\hline One50 AR1 & 107 & $a b$ & 99 & $\mathrm{bg}$ & 99 & $\mathrm{~cd}$ & 107 & $\mathrm{bc}$ & 104 & bd & 103 & bd \\
\hline Bronsyn SE & 84 & $\mathrm{i}$ & 98 & bg & 102 & ad & 106 & bd & 106 & bd & 103 & bd \\
\hline Arrow WE & 103 & be & 105 & $\mathrm{a}$ & 106 & $a b$ & 98 & $\mathrm{dg}$ & 98 & ef & 102 & bd \\
\hline Impact SE & 104 & be & 90 & hi & 99 & bd & 104 & be & 107 & ad & 101 & bd \\
\hline Extreme AR1 & 97 & eg & 105 & $a b$ & 98 & ce & 103 & be & 98 & ef & 101 & bd \\
\hline Alto WE & 99 & $\mathrm{df}$ & 96 & eh & 101 & ad & 102 & bf & 100 & $d f$ & 101 & bd \\
\hline Revolution AR1 & 104 & be & 100 & af & 101 & bd & 97 & eg & 98 & ef & 100 & $\mathrm{~cd}$ \\
\hline Bronsyn AR1 & 93 & fh & 100 & af & 100 & bd & 101 & $c f$ & 103 & ce & 100 & $d$ \\
\hline Commando WE & 93 & gh & 107 & a & 100 & bd & 92 & $\mathrm{~g}$ & 95 & $f$ & 97 & de \\
\hline Commando AR1 & 84 & $\mathrm{i}$ & 97 & $\mathrm{dh}$ & 100 & bd & 93 & g & 87 & g & 94 & e \\
\hline Tetraploid cultiva & & & & & & & & & & & & \\
\hline Bealey NEA2 & 106 & ac & 95 & gh & 97 & de & 104 & bd & 104 & bd & 100 & $\mathrm{~cd}$ \\
\hline Banquet II Endo5 & 104 & be & 96 & fh & 96 & de & 105 & bd & 103 & ce & 100 & $\mathrm{~cd}$ \\
\hline Banquet SE & 108 & $a b$ & 89 & $\mathrm{i}$ & 94 & e & 95 & $\mathrm{fg}$ & 97 & $f$ & 95 & e \\
\hline Quartet SE & 74 & $\mathrm{j}$ & 77 & j & 88 & $f$ & 73 & $\mathrm{~h}$ & 81 & g & 79 & $f$ \\
\hline Mean (kgDM/ha) & \multicolumn{2}{|c|}{1067} & \multicolumn{2}{|c|}{1999} & \multicolumn{2}{|c|}{3595} & \multicolumn{2}{|c|}{4094} & \multicolumn{2}{|c|}{2545} & \multicolumn{2}{|c|}{13322} \\
\hline Wald test & \multicolumn{2}{|c|}{$<0.001$} & \multicolumn{2}{|c|}{$<0.001$} & \multicolumn{2}{|c|}{$<0.001$} & \multicolumn{2}{|c|}{$<0.001$} & \multicolumn{2}{|c|}{$<0.001$} & \multicolumn{2}{|c|}{$<0.001$} \\
\hline LSD 5\% & \multicolumn{2}{|c|}{9.2} & \multicolumn{2}{|c|}{8.3} & \multicolumn{2}{|c|}{7.9} & \multicolumn{2}{|c|}{10.8} & \multicolumn{2}{|c|}{9.9} & \multicolumn{2}{|c|}{7.3} \\
\hline
\end{tabular}

${ }^{*}$ Endophyte strain is given after cultivar name. SE = Standard or wild-type endophyte. WE= without (nil) endophyte. 
ranging from a fall of $3.54 \mathrm{t} \mathrm{DM} / \mathrm{ha} /$ year in the Waikato trials to a gain of $1.15 \mathrm{t}$ DM between Years 1 and 3 in the Taranaki trials (Table 3). Interestingly, no cultivar $\times$ year interaction was detected, indicating that all cultivars in the trials displayed a similar yield decline with time. The variation between regions in rate of yield decline is possibly linked to summer moisture deficit stress as indicated by regional rainfall statistics in Table 3, but this point needs further investigation.
The interaction between region and cultivar was also statistically significant $(\mathrm{P}<0.05)$ and this too warrants further investigation.

\section{Persistence}

Pasture persistence has a significant effect on the performance of both dairy (Brazendale et al. 2011) and sheep and beef systems (Stevens 2011). The ability of perennial ryegrass to persist across the wide range

Table 3: $\quad$ Analysis of regional mean yield (tonnes $\mathrm{DM} / \mathrm{ha}$ ) and change in yield over time for a subset of 234 annual mean yields of 10 cultivars in 5 regions. Cultivars included in the analysis were 'Alto', 'Arrow', 'Bealey', 'Bronsyn', 'Commando', 'Extreme', 'Matrix', 'One50', 'Revolution' and 'Trojan.'

\begin{tabular}{llllll}
\hline Region & Year 1 & Year 2 & Year 3 & $\begin{array}{l}\text { Change year 1 } \\
\text { to year 3 }\end{array}$ & $\begin{array}{l}\text { Nov }- \text { March } \\
\text { rainfall }\end{array}$ \\
Waikato & 18.8 & 18.7 & 13.5 & -5.34 & $395 \mathrm{~mm}$ \\
Taranaki & 13.8 & 14.3 & 14.9 & 1.15 & $629 \mathrm{~mm}$ \\
Manawatu & 13.9 & 11.0 & 10.8 & -3.06 & $354 \mathrm{~mm}$ \\
Canterbury & 12.0 & 12.1 & 11.2 & -0.79 & $214 \mathrm{~mm}^{*}$ \\
Southland & 13.0 & 12.9 & 11.3 & -1.70 & $489 \mathrm{~mm}^{*}$
\end{tabular}

+ Mean for 2003 - 2011 years (Taranaki) and 2002 - 2011 (other regions). ' Canterbury trials were irrigated.

Table 4: $\quad$ Analysis of ryegrass ground cover at the end of trials for: (1) nine North Island trials from 2003-11, and; (2) two Newstead trials run at the time of 2007/08 drought. Significance lettering given for $5 \%$ LSD. (T) = tetraploid. For the North Island data, a minimum of three scores were necessary for a cultivar to be included, except where marked with * (two scores).

\begin{tabular}{|c|c|c|c|c|c|}
\hline \multirow{2}{*}{$\begin{array}{l}\text { Cultivar } \\
\text { Tolosa NEA2* }\end{array}$} & \multicolumn{2}{|c|}{$\begin{array}{l}\text { Ground cover \% } \\
\text { North Island trials }\end{array}$} & \multirow{2}{*}{$\begin{array}{l}\text { Cultivar } \\
\text { Arrow AR1 }\end{array}$} & \multicolumn{2}{|c|}{$\begin{array}{l}\text { Ground cover \% } \\
\text { Newstead trials }\end{array}$} \\
\hline & 71 & $\mathrm{a}$ & & 87 & a \\
\hline Arrow AR1 & 69 & $a b$ & Trojan NEA2 & 84 & $a b$ \\
\hline Trojan NEA2 & 68 & ac & Alto AR1 & 84 & $a b$ \\
\hline Bronsyn NEA6 & 68 & ac & Bealey NEA2 (T) & 83 & $a b$ \\
\hline Matrix SE & 67 & ac & Banquet SE (T) & 83 & $a b$ \\
\hline Bealey NEA2 (T) & 67 & ac & One50 AR1 & 81 & ac \\
\hline Banquet SE (T) & 66 & ac & Bronsyn AR1 & 76 & bd \\
\hline One50 AR1 & 66 & ad & Arrow SE & 70 & $\mathrm{~cd}$ \\
\hline Alto AR1 & 66 & ad & Commando AR1 & 70 & $\mathrm{~cd}$ \\
\hline Bronsyn AR1 & 66 & ad & Revolution AR1 & 67 & de \\
\hline Commando AR1 & 65 & ad & Extreme AR37 & 59 & ef \\
\hline Impact SE & 65 & ad & Extreme AR6 & 55 & $\mathrm{fg}$ \\
\hline Bronsyn SE & 65 & ad & Banquet II Endo5 (T) & 55 & $\mathrm{fg}$ \\
\hline Arrow SE & 65 & bd & Extreme AR1 & 50 & $g$ \\
\hline Alto SE & 64 & bd & $\mathrm{F}$ test & \multicolumn{2}{|c|}{$<0.001$} \\
\hline Revolution AR1 & 62 & $\mathrm{ce}$ & LSD 5\% & \multicolumn{2}{|c|}{10.0} \\
\hline Banquet II Endo5 (T) & 62 & $\mathrm{ce}$ & CV\% & \multicolumn{2}{|c|}{8.0} \\
\hline Extreme AR1* & 59 & df & & & \\
\hline Extreme AR37* & 56 & ef & & & \\
\hline Extreme AR6 & 54 & $f$ & & & \\
\hline Wald test & \multicolumn{2}{|c|}{$<0.001$} & & & \\
\hline LSD 5\% & \multicolumn{2}{|c|}{7.5} & & & \\
\hline CV\% & \multicolumn{2}{|c|}{11.3} & & & \\
\hline
\end{tabular}


of conditions in which it is used has been questioned (Fraser 1994; Thom et al. 1998; Easton et al. 2011). Persistence issues have become particularly prominent in the upper North Island in the last 4 years, where factors including summer moisture deficits and black beetle (Heteronychus arator) attack have placed pressure on perennial ryegrass pastures (Kelly et al. 2011; Tozer et al. 2011).

Point analysis to assess the ground cover by ryegrass at the end of each 3-year trial is the prescribed method to assess ryegrass persistence in seed industry protocols (NZPBRA 2010). These data are presented in Table 4 in two ways. Firstly, combined for all the North Island trials, and secondly, for just the two Waikato trials located at Newstead which suffered a severe " 1 in 100 year drought" in 2007/08.

The North Island trials are presented because they were conducted under more difficult conditions, with

Table 5: Analysis of 18 ryegrass plant pulling scores from six trials from 2003-11, on a 1 to 9 basis where 9 $=$ no plant pulling. Significance lettering given for $5 \%$ LSD. Cultivars must have a minimum of three scores to be included. $(T)=$ tetraploid.

\begin{tabular}{|c|c|c|}
\hline \multirow{2}{*}{$\begin{array}{l}\text { Cultivar } \\
\text { Bealey NEA2 (T) }\end{array}$} & \multicolumn{2}{|c|}{$\begin{array}{l}\text { Average plant pulling } \\
\text { score }{ }^{*}\end{array}$} \\
\hline & 8.0 & a \\
\hline Bronsyn SE & 8.0 & a \\
\hline Bronsyn NEA6 & 7.9 & $a b$ \\
\hline Banquet SE (T) & 7.7 & $a b$ \\
\hline One50 AR1 & 7.7 & ac \\
\hline Bronsyn AR1 & 7.6 & ac \\
\hline Alto SE & 7.5 & ad \\
\hline Arrow SE & 7.3 & $\mathrm{ad}$ \\
\hline Trojan NEA2 & 7.2 & $\mathrm{ae}$ \\
\hline Commando AR1 & 7.0 & bf \\
\hline Commando WE & 7.0 & bf \\
\hline Impact SE & 6.9 & $\mathrm{cf}$ \\
\hline Alto AR1 & 6.9 & $\mathrm{cf}$ \\
\hline Arrow AR1 & 6.8 & df \\
\hline Matrix SE & 6.4 & ef \\
\hline Banquet II Endo5 (T) & 6.2 & $f$ \\
\hline Extreme AR6 & 5.3 & g \\
\hline Extreme AR37 & 5.1 & $g$ \\
\hline Revolution AR1 & 5.0 & g \\
\hline Extreme AR1 & 4.4 & $g$ \\
\hline $\mathrm{F}$ test & \multicolumn{2}{|c|}{$<0.001$} \\
\hline LSD (5\%) & \multicolumn{2}{|c|}{0.8} \\
\hline CV\% & \multicolumn{2}{|c|}{10.2} \\
\hline
\end{tabular}

* Data are combined from six trials: 2004 Whareroa (4 scores), 2005 Newstead (5 scores), 2005 Massey University (1 score), 2005 Burnham (2 scores), 2006 Whareroa (1 score) and 2007 Newstead (5 scores). warmer temperatures and less reliable summer rainfall than the South Island sites. Despite this, ground cover differences between cultivars in the North Island were small.

The Newstead trial results in Table 4 also show few differences. Most cultivars containing AR1 endophyte had high ground covers, at odds with what was happened on many farms through this drought period, including farmlet trials at the same site (Thom 2010).

The results from point analysis at the end of these types of small plot trials do not, therefore, appear to assess the persistence of ryegrass cultivars well enough to be representative of a wide range of farms. There are three suggested reasons for this. Firstly, there is careful pasture management of the trials, which is not always replicated on commercial farms, particularly through periods of low pasture growth. Secondly, the plots are sown and kept as pure ryegrass swards with herbicide applications, so are artificially free of white clover and

Table 6: Analysis of seven rust scores from four trials from 2003-11, on a 1 to 9 basis where $9=$ no rust. Significance lettering given for 5\% LSD. Cultivars must have a minimum of two scores to be included. (T) $=$ tetraploid

\begin{tabular}{|c|c|c|}
\hline \multirow{2}{*}{$\begin{array}{l}\text { Entry } \\
\text { Extreme AR1 }\end{array}$} & \multicolumn{2}{|c|}{ Average rust score* } \\
\hline & 9.0 & a \\
\hline One50 AR1 & 9.0 & a \\
\hline Extreme AR37 & 8.7 & a \\
\hline Bealey NEA2 (T) & 8.0 & $a b$ \\
\hline Commando AR1 & 7.8 & $a b$ \\
\hline Extreme AR6 & 7.8 & ac \\
\hline Arrow AR1 & 7.1 & bd \\
\hline Bronsyn NEA6 & 6.7 & $\mathrm{ce}$ \\
\hline Trojan NEA2 & 6.5 & cf \\
\hline Alto AR1 & 6.5 & cf \\
\hline Alto SE & 6.2 & df \\
\hline Bronsyn AR1 & 6.1 & df \\
\hline Matrix SE & 6.1 & df \\
\hline Revolution AR1 & 5.7 & $\mathrm{dg}$ \\
\hline Bronsyn SE & 5.6 & eg \\
\hline Banquet II Endo5 (T) & 5.3 & fg \\
\hline Impact SE & 5.1 & $g$ \\
\hline Quartet SE (T) & 4.9 & g \\
\hline Banquet SE (T) & 4.6 & $g$ \\
\hline F test & $<0.001$ & \\
\hline LSD 5\% & 1.1 & \\
\hline CV\% & 12.9 & \\
\hline
\end{tabular}

* Data are combined from four trials: 2003 Massey (2 scores), 2004 Whareroa (1 score), 2005 Newstead (2 score) and 2007 Massey University (2 scores). 
weed ingression common with poor persistence (Tozer et al. 2011). Thirdly, the trials only run for 3 years.

To better assess persistence new evaluation protocols are required to more closely simulate commercial farming conditions, with trials run over a longer time period. One suggestion is to test cultivars in an additional trial series on commercial farms, using mixed swards, on sites known to provide a challenge to perennial ryegrass growth and survival.

\section{Plant pulling}

Ryegrass plant "pulling" occurs in some areas of New Zealand, particularly throughout northern North Island peat and light ash soils under cattle grazing (Thom et al. 2003) and differences in susceptibility exist between cultivars (Thom et al. 1996).

In the trials, plant pulling was distinguished by visual scores of plots, with clear differences in resistance shown between cultivars (Table 5). Plant pulling and ground cover scores for the North Island trials (Table 4) were correlated $\left(R^{2}=0.76\right)$, indicating that plant loss through pulling caused sustained reductions in ryegrass plant/tiller density .

Prestidge et al. (1989) suggested that endophyte strain might affect plant pulling, however, in this case cultivars with multiple endophyte strains ('Alto', 'Arrow', 'Bronsyn', 'Commando' and 'Extreme') exhibited similar levels of plant pulling irrespective of endophyte strain.

Similarly, no relationship between plant pulling and heading date was apparent, with mid-season and late heading cultivars showing both high and low levels of pulling.

\section{Rust}

Crown rust (Puccinia coronata) is a common pathogen which reduces both the photosynthetic leaf area and the acceptability of foliage to livestock (Easton et al. 1989). Crown rust only occurred in the North island trials where higher temperatures and humidity favour its development (Latch \& Lancashire 1966), and no rust was seen in Canterbury trials, where stem rust (Puccinia graminis) is more prevalent (Easton et al. 1989).

Ryegrass cultivars can be selected for improved rust resistance (Easton et al. 2002) and significant cultivar differences were identified by visual scoring of plots (Table 6).

Endophyte strain appeared to have little effect on rust resistance, as those cultivars with multiple endophyte strains ('Alto', 'Bronsyn' and 'Extreme') were not significantly different in rust resistance across strains. Similarly, heading date appeared to have no effect with late and mid-season cultivars displaying both high and low levels of rust resistance.

\section{Conclusion}

The small plot trials in this programme generated useful comparative information on the potential DM yield and seasonal growth pattern of a range of perennial ryegrass cultivars and breeding lines. They also identified significant differences between ryegrasses in rust resistance and, under dairy cow grazing, in susceptibility to plant pulling.

However, there were limitations associated with the trial methods, the most notable being that they did not reflect the persistence of different cultivars that was seen on many farms in the northern North Island through the same period.

Different trialling methods are needed to better assess ryegrass persistence. One suggestion is to establish "mirror image" trial series on commercial farms, to better simulate "real" farm conditions, sown with ryegrass and white clover, with more typical herbicide usage, and trials run for longer than the industry-norm of 3 years. Persistence differences should emerge more quickly if sites known to provide a challenge to perennial ryegrass growth and survival were chosen for trials.

A better assessment tool for persistence is also needed. One-off point analysis of ryegrass ground cover, as prescribed by industry protocols, is of limited value. Also ryegrass ground cover interacts with plant structure, such that cultivars with fewer, larger tillers tend to have lower ground cover (Neuteboom et al. 1988) but their yield may be similar to cultivars with a greater density of smaller tillers (Bahmani et al. 2001).

\section{REFERENCES}

Bahmani, I.; Thom, E.R.; Matthew, C.; Lemaire, G. 2001. Productivity of grazed perennial ryegrass dairy pastures from different ecotypes under nitrogen and irrigation treatments. New Zealand Journal of Agricultural Research 44: 123-133.

Brazendale, R.; Bryant, J.R.; Lambert, M.G.; Holmes, C.W.; Fraser, T. 2011. Pasture persistence: how much is it worth? Pasture Persistence Symposium, Grassland Research and Practice Series No.15: 3-6. Bryant, J.; Chapman, D.; McMillan, W.; Thorrold, B.; Willocks, M.; Green, D.; Kerr, G.; Judson, G.; Cookson, T.; Edwards, G. 2012. Valuing the forage you grow: Economic indices for pasture cultivars. Proceedings of the South Island Dairy Event: pp 28-34.

Chapman, D.F.; Bryant, J.R.; McMillan, W.H. 2012. Economic values for evaluating pasture traits. Proceedings of the New Zealand Grassland Association 74: 209-216.

Clark, D.A.; Matthew, C.; Crush J.R. 2001. More feed for New Zealand dairy systems. Proceedings of the New Zealand Grassland Association 63: 283-288. 
Corkill, L. 1949. Pasture improvement in New Zealand. Empire Journal of Experimental Agriculture 17: 157-169.

Crush, J.R.; Woodward, S.L.; Eerens, J.P.J.; Macdonald, K.A. 2006. Growth and milksolids production in pastures of older and more recent ryegrass and white clover cultivars under dairy grazing. New Zealand Journal of Agricultural Research 49: 119-135.

Easton, H.S.; Cooper, B.M.; Fraser, T.J.; Widdup, K.H. 1989. Crown rust on perennial ryegrass in field trials. Proceedings of the New Zealand Grassland Association 50: 253-254.

Easton, S.; Baird, D.; Baxter, G.; Cameron, N.E.; Hainsworth, R.; Johnston, C.; Kerr, G.A.; Lyons, T.; McCabe, R.; Nichol, W.; Norriss, M.G.; Stewart, A.V.; Thom, E.R. 1997. Annual and hybrid ryegrass cultivars in New Zealand. Proceedings of the New Zealand Grassland Association 59: 239-243.

Easton, H.S.; Baird, D.B.; Cameron, N.E.; Kerr, G.A.; Norriss, M.G.; Stewart, A.V. 2001. Perennial ryegrass cultivars: herbage yield in multi-site plot trials. Proceedings of the New Zealand Grassland Association 63: 183-188.

Easton, H.S.; Amyes, J.M.; Cameron, N.E.; Green, R.B.; Kerr, G.A.; Norriss M.G.; Stewart, A.V. 2002. Pasture plant breeding in New Zealand: where to from here? Proceedings of the New Zealand Grassland Association 64: 173-179.

Easton, H.S.; Kerr, G.A.; Stewart, A.V. 2011. Ryegrass in pastures - breeding for resilience. Pasture Persistence Symposium, Grassland Research and Practice Series No.15: 139-148.

Edwards, G.R.; Lucas, R.J.; Johnston, M.R. 1993. Grazing preference for pasture species by sheep is affected by endophyte and nitrogen fertility. Proceedings of the New Zealand Grassland Association 55: 137-141.

Fraser, T.J. 1994. Persistence of dryland pasture species in mixed swards in Canterbury. Proceedings of the New Zealand Grassland Association 56: 77-79.

Hume, D.E.; Ryan, D.L.; Cooper, B.M.; Popay, A.J. 2007. Agronomic performance of AR37-infected ryegrass in northern New Zealand. Proceedings of the New Zealand Grassland Association 69: 201205.

Hunt, W.F.; Easton, H.S. 1989. Fifty years of ryegrass research in New Zealand. Proceedings of the New Zealand Grassland Association 50: 11-23.

Kelly, S.; Smith, E.; Brazendale, R. 2011. Pasture Renewal in the Waikato and Bay of Plenty regions: An overview of farmer practice, experience and attitudes. Pasture Persistence Symposium, Grassland Research and Practice Series 15: 21-24.
Kerr, G.A. 1987. Evaluation of four perennial ryegrass cultivars in New Zealand. Proceedings of the New Zealand Grassland Association 48: 137-141.

Latch, G.C.M.; Lancashire, J.A. 1966. Some effects of crown rust (Puccinia coronata Corda) on the growth of two ryegrass varieties in New Zealand. New Zealand Journal of Agricultural Research 9: 628-640.

Lee, J.M.; Matthew, C.; Thom, E.R.; Chapman, D.F. 2012. Perennial ryegrass breeding in New Zealand: a dairy industry perspective. Crop and Pasture Science 63: 107-127.

McEvoy, M.; O’Donovan, M.; Shalloo, L. 2011. Development and application of an economic ranking index for perennial ryegrass cultivars. Journal of Dairy Science 94: 1627-1639.

Neuteboom, J. H.; Lantinga, E. A.; Wind, K. 1988. Tillering characteristics of diploid and tetraploid perennial ryegrass. Proceedings of the 12th General Meeting of the European Grassland Federation, Dublin, Ireland: 498-503.

NZPBRA. 2010. National Forage Variety Trials (NFVT) - Trial Protocol July 2010. New Zealand Plant Breeding and Research Organisation.

O'Donovan, M.; Delaby, L. 2005. A comparison of perennial ryegrass cultivars differing in heading date and ploidy and spring calving dairy cows grazed at two different stocking rates. Animal Research 54: $337-350$.

Parsons, A.J.; Edwards, G.R.; Newton, P.C.D.; Chapman, D.F.; Caradus, J.R.; Rasmussen, S.; Rowarth, J.S. 2011. Past lessons and future prospects: plant breeding for yield and persistence in cool temperate pastures. Grass and Forage Science 66: 153-172.

Pennell, C.G.L.; Easton, H.S.; Goold, G. J.; Hay, R.J.M.; Hickey, M. J.; Hoglund, J.H.; Lyons, T.B.; McCallum, D.A.; Paewai, H.R.T.; Stevens, D.R.; Thomson, N.A.; Woods, P.W. 1990. 'Grasslands Pacific' perennial ryegrass: performance at several sites. Proceedings of the New Zealand Grassland Association 52: 151-155.

Popay, A.J.; Hume, D.E. 2011. Endophytes improve ryegrass persistence by controlling insects. Pasture Persistence Symposium, Grassland Research and Practice Series No.15: 149-156.

Popay, A.J.; Hume, D.E.; Davis, K.L.; Tapper, B.A. 2003. Interactions between endophyte (Neotyphodium spp.) and ploidy in hybrid and perennial ryegrass cultivars and their effects on Argentine stem weevil (Listronotus bonariensis). New Zealand Journal of Agricultural Research 46: 311-319. 
Prestidge, R.A.; van der Zijpp, S.; Gault, P. 1989. Effect of Argentine stem weevil on vigour of 'Grasslands Roa' tall fescue under dairying in the Waikato. New Zealand Journal of Agricultural Research 32: 291297.

Radcliffe, J.E.; Mountier, N.S. 1964. Problems in measuring pasture composition in the field. Part 1. Discussion of general problems and some considerations of the point method. New Zealand Journal of Botany 2: 90-97.

Stevens, D.R. 2011. Managing sheep and beef farm systems - where does pasture persistence fit in? Pasture Persistence Symposium, Grassland Research and Practice Series 15: 191-198.

Thom, E.R. 2010. AR37 endophyte research - project comes to a close. Inside Dairy, February: 7-9.

Thom, E.R.; van Vught, V.T.; McCabe R.J. 1996. Growth and persistence of perennial ryegrass lines with different tolerances to "pulling" during grazing. Proceedings of the New Zealand Grassland Association 58: 67-72.

Thom, E.R.; Waugh, C.D.; McCabe, R.J. 1998. Growth and persistence of perennial and hybrid ryegrasses when grazed by dairy cows in the central Waikato region of New Zealand. New Zealand Journal of Agricultural Research 41: 477-486.
Thom, E.R.; Burggraaf, V.T.; Watts, R.J.; Hooper, R.J. 2003. Relationship of tillering and morphological characteristics of two perennial ryegrass lines to "pulling" when grazed by dairy cows. New Zealand Journal of Agricultural Research 46: 15-25.

Tozer, K.N.; Cameron, C.A.; Thom, E.R. 2011. Pasture persistence: farmer observations and field measurements. Pasture Persistence Symposium, Grassland Research and Practice Series No.15: 25-30.

Williams, W.M.; Easton, H.S.; Jones, C.S. 2007. Future options and targets for pasture plant breeding in New Zealand. New Zealand Journal of Agricultural Research 50: 223-248.

Woodfield, D.R. 1999. Genetic improvements in New Zealand forage cultivars. Proceedings of the New Zealand Grassland Association 61: 3-7.

Woodward, S.L.; Macdonald, K.A.; Carter, W.A.; Eerens, J.P.J.; Crush, J.R. 2001. Milksolids production from different combinations of perennial ryegrass and white clover cultivars: II Milksolids production and farm profitability. Proceedings of the New Zealand Grassland Association 63: 97-102.

Wratt, G.S.; Smith, H.C. 1983. Plant Breeding in New Zealand. Butterworths, DSIR, Wellington. 309 pp. 
